\title{
Autonomous Wireless System for Robust and Efficient Inductive Power Transmission to Multi-Node Implants
}

\author{
Peilong Feng*† and Timothy G. Constandinou*†‡ \\ *Department of Electrical and Electronic Engineering, Imperial College London, SW7 2BT, UK \\ ${ }^{\dagger}$ Centre for Bio-Inspired Technology, Institute of Biomedical Engineering, Imperial College London, SW7 2AZ, UK \\ ${ }^{\ddagger}$ Care Research \& Technology Centre, UK Dementia Research Institute, UK \\ Email: \{peilong.feng14, t.constandinou\}@imperial.ac.uk
}

\begin{abstract}
A number of recent and current efforts in brain machine interfaces are developing millimetre-sized wireless implants that achieve scalability in the number of recording channels by deploying a distributed 'swarm' of devices. This trend poses two key challenges for the wireless power transfer: (1) the system as a whole needs to provide sufficient power to all devices regardless of their position and orientation; (2) each device needs to maintain a stable supply voltage autonomously. This work proposes two novel strategies towards addressing these challenges: a scalable resonator array to enhance inductive networks; and a selfregulated power management circuit for use in each independent mm-scale wireless device. The proposed passive 2-tier resonant array is shown to achieve an $11.9 \%$ average power transfer efficiency, with ultra-low variability of $1.77 \%$ across the network.

The self-regulated power management unit then monitors and autonomously adjusts the supply voltage of each device to lie in the range between $1.7 \mathrm{~V}-1.9 \mathrm{~V}$, providing both low-voltage and over-voltage protection.
\end{abstract}

\section{INTRODUCTION}

A number of different wireless interface techniques have been applied to implantable medical devices for transmitting power and communicating data, such as ultrasonic [1]-[4], electromagnetic [5]-[14], optical [15]-[17]. A key objective of implementing any scheme for power transmission to an implant is to avoid the need for wires, particularly percutaneous thus reducing the risk of infection. Much of the research literature focuses on improving the power transfer efficiency whilst also ensuring biological safety (e.g. maintaining thermal dissipation to an acceptable level) [18]-[20]. These schemes are typically designed for a single (or limited) number of implanted devices, and rely on relatively precise positioning and thus good alignment between transmitter and receiver coils that themselves are relatively large [21]. This is therefore not directly applicable to a scenario with multiple, freely-position implants that all need to be simultaneously powered and are of millimetre-scale [22]-[24].

Wireless power transfer for invasive brain machine interface applications are particularly challenging where the distances and alignments between external transmitter (Tx) and implantable receiver $(\mathrm{Rx})$ vary based on cerebral cortex. This results in different coupling coefficients to each of the $\mathrm{Rx}$ coils that in turn results in a widely variable, and unpredictable induced voltage on the secondary coil. Furthermore, this is not purely a static setup that can be calibrated, or configured after implantation, as the micromotion of brain itself (i.e. within the

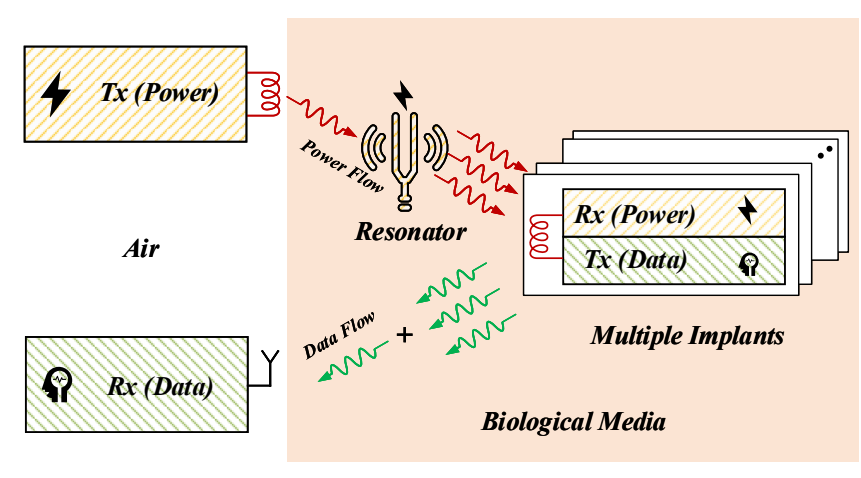

Fig. 1. Simplified system architecture for proposed wireless information and power transmission (WIPT) scheme.

skull) can result is the received voltage fluctuating over time. Adjusting the Tx output power can compensate for secondary voltage fluctuations for a single $\mathrm{Rx}$ device. This however is not a practical solution in systems with multiple $\mathrm{Rx}$ devices.

This paper presents a wireless infrastructure that uses a resonator-array to enhance the inductive coupling to freelyfloating implantable devices for achieving wireless information and power transfer (WIPT). The paper is organised as follows: the wireless concept is first described in Section II; the enhancement to coverage (and significantly reduced sensitivity to misalignment) is then demonstrated through comparing this to a number of typical schemes, described in Section III; Section IV then presents the self-regulated power management unit to tackle uneven spatial PTE by automatically tuning the self-resonant frequency at each secondary node (i.e. freelyfloating implant).

\section{SYSTEM OVERVIEW}

The proposed WIPT system architecture is shown in Fig. 1. This fundamentally facilitates a Power In \& Data Out function. The downlink is used to transmit power wirelessly from a single external transceiver to multiple freely-positioned implants through the relay resonator. In a BMI application, each of these implants will contain instrumentation circuits to record neural activity from electrodes. The uplink is then used to send data from each of these freely-positioned implants to the external device. The external device will thus collect data recorded across a distributed network of implants. 
The proposed WIPT scheme provides three key features for a distributed wireless power transmission and data communication system:

1) Enhanced power transfer efficiency: The maximum inductively coupled power transmission efficiency occurs on the same resonant frequency of Tx and Rx LC tank. At the resonant condition, electromagnetic energy is coupled to the Rx LC tank through evanescent waves [25]. However, the amplitude of the evanescent waves can be enhanced by using a resonator, which results in coupling coefficient enhancement between the Tx and Rx, and eventually improves the PTE of the wireless power transfer (WPT) system based on resonant inductive coupling mechanism [6].

2) Uniform power coverage: Power coverage is determined by the magnetic flux density $B$. The resonator evenly redistributes the magnetic field density over a wider area than that of the $\mathrm{Tx}$ coil. Therefore, a Rx coil positioned outside the 'coverage' of the Tx alone can obtain sufficient power.

3) Low power consumption: The system uses back-scatter via the power link to modulate data and achieve the uplink without requiring any additional data carrier [26].

From a biological perspective, this scheme provides three key features: (1) reduced risk of infection risk due to no need for percutaneous wiring; (2) reduced tissue response and thus improved reliability due to avoiding the need for any tethers or implanted leads (e.g. [27], [28]); (3) for distributed implants, the avoidance of thermal 'hotspots' due to a uniformly distributed field.

\section{Wireless Power Transfer Schemes}

The first step in designing a WPT system for an distributed implant network, is to select the which coil configuration is most appropriate. In this section, we compare the following 4 schemes in terms of PTE and power coverage: 2-coil, 3-coil, 3 -coil with a 1-tier resonator array, and 4-coil with a 2-tier resonator array.

\section{A. Power Transfer Efficiency of four WPT Schemes}

1) 2-coil: Wireless power transmission conventionally uses a 2-coil inductive link. However, for coil pairs with a relatively large separation relative to the coil diameters, this suffers from a low power transfer efficiency due to the weak coupling coefficient between Tx and Rx coils. Furthermore, a 2-coil configuration requires near perfect alignment between $\mathrm{Tx}$ and $\mathrm{Rx}$ coil. Its power transfer efficiency is thus highly sensitive to positional and angular misalignment [29]. This is because it utilises a single Tx (primary) coil that generates a centrally focused electromagnetic field that is undesirable if requiring uniform coverage.

Fig. 2(a.1) shows a simplified equivalent schematic of 2coil inductive link. This contains an external voltage source $V_{s}$, source impedance $R_{s}$ at the primary side, and load impedance $R_{L}$ at the secondary side. According to reflected load theory [30], the PTE of a 2-coil inductive link can be expressed as

$$
P T E_{2-c o i l}=\frac{k_{14}^{2} Q_{1} Q_{4 L}}{1+k_{14}^{2} Q_{1} Q_{4 L}} \cdot \frac{Q_{4 L}}{Q_{L}}
$$
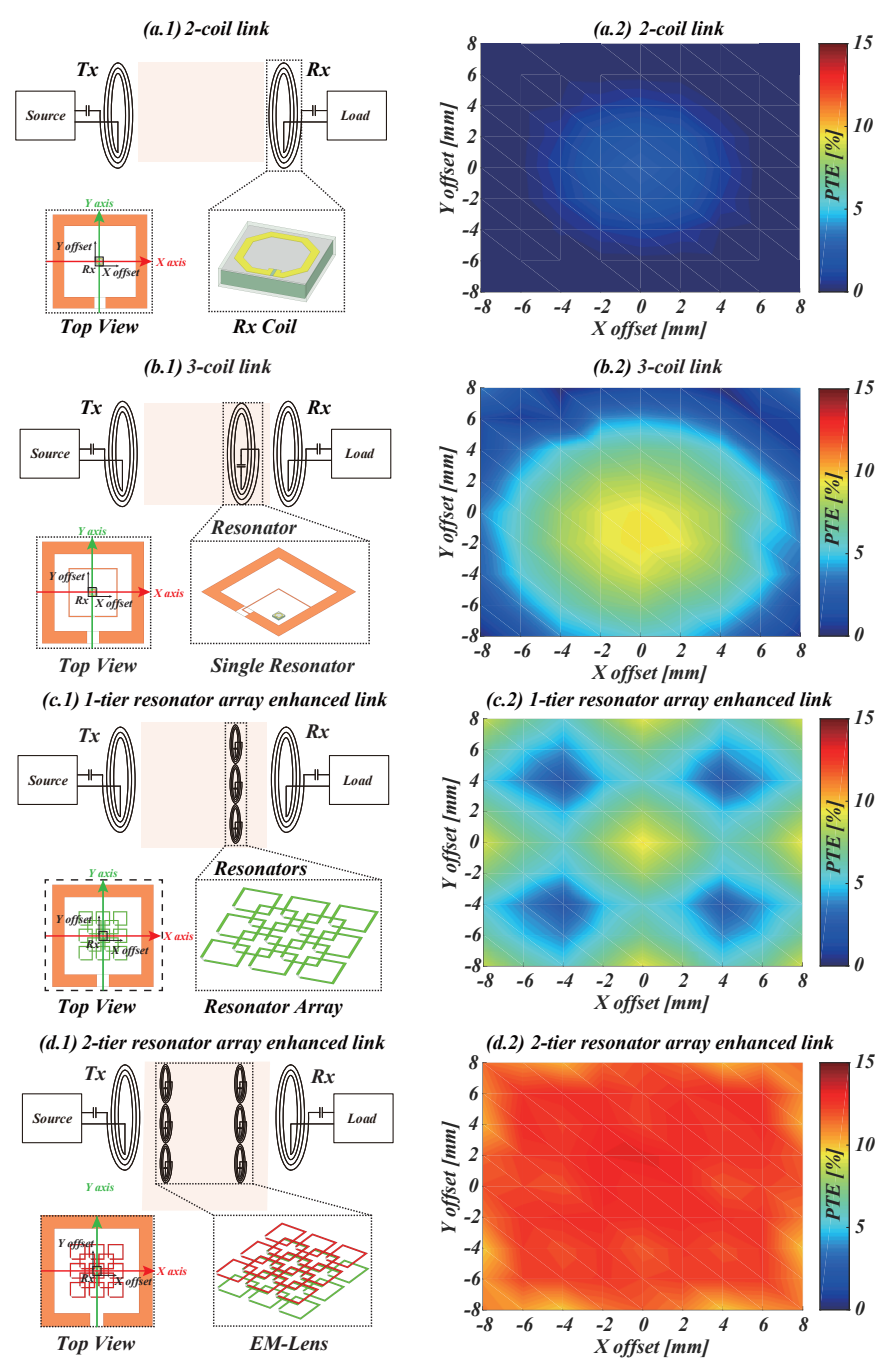

Fig. 2. Simulation models and corresponding simulated results of power transfer efficiency across $16 \mathrm{~mm} \times 16 \mathrm{~mm}$ area: (a) 2-coil; (b) 3-coil; (c) 3 -coil with 1-tier resonator array; and (d) 4-coil with 2-tier resonator array.

where the quality factor $Q_{1}$ of the primary $\mathrm{L}_{1} \mathrm{C}_{1}$ tank and loaded quality factor $Q_{4 L}$ of secondary $\mathrm{L}_{2} \mathrm{C}_{2}$ tank are written as: $Q_{1}=\omega L_{1} / R_{1}, Q_{4 L}=Q_{4} Q_{L} / Q_{4}+Q_{L}$, where $Q_{4}=$ $\omega L_{4} / R_{4}, Q_{L}=R_{L} / \omega L_{4}$.

2) 3-coil: The 3-coil configuration introduce a resonator $L_{3} C_{3}$ (i.e. third coil) between the primary $L_{1}$ and secondary $L_{2}$ coils (of the 2-coil configuration). The expression for the PTE of the 3-coil configurations consists of two terms:

$$
\begin{aligned}
& P T E_{3-\text { coil }}=P T E_{14}+P T E_{13} \cdot P T E_{34} \\
& =P T E_{2-\text { coil }}+P T E_{13} \cdot P T E_{34}
\end{aligned}
$$

where the first term $P T E_{14}$ is the same as the 2-coil link in Eq. 1, describing the PTE from the primary to secondary side. The second term $P T E_{13} \cdot P T E_{34}$ represents the coupling through the resonance coil. The PTE from primary coil $L_{1}$ to resonator $L_{3}$ is defined as [30]:

$$
P T E_{13}=\frac{k_{13}^{2} Q_{1} Q_{3}}{1+k_{13}^{2} Q_{1} Q_{3}+k_{34}^{2} Q_{3} Q_{4 L}}
$$


and the PTE from resonator $L_{3}$ to secondary inductor $L_{4}$ is defined as:

$$
P T E_{34}=\frac{k_{34}^{2} Q_{3} Q_{4 L}}{1+k_{34}^{2} Q_{3} Q_{4 L}} \cdot \frac{Q_{4 L}}{Q_{L}}
$$

The PTE of the 3-coil link is therefore greater than that of 2-coil link $\left(P T E_{3-\text { coil }}>P T E_{2-\text { coil }}\right)$.

3) 3-coil with 1-tier resonator array: Here the single resonator $L_{3}$ within a simple 3-coil configuration is replaced by an array of coils $L_{3 j}$, as shown in Fig. 2(c.1). Similar to the 3-coil configuration, its PTE is also composed of two parts:

$$
P T E_{1-\text { tier }}=P T E_{14}+\sum_{j=1}^{n} P T E_{13 j} \cdot P T E_{3 j 4}
$$

where $P T E_{13 j}$ and $P T E_{3 j 4}$ represent the PTE of $L_{1}$ and $L_{4}$ coupled with each resonator unit $L_{3 j}$, and their equations are same as Eq. 3 and 4.

4) 4-coil with 2-tier resonator array: The 2-tier resonator array implements the two intermediate coils within a 4-coil configuration using two sets of resonators $L_{2 i}, L_{3 j}$. The difference between this configuration and the EM-Lens previously reported in [14] is that the wired connections between each resonator unit have been removed. Also, the upper and lower resonator arrays here are independent with no connections between the two tiers. Thus, the self-resonant frequency (SRF) deviation of each resonator unit caused by internal wired connections can be eliminated, which results in PTE and coverage improvement. The PTE can here be defined as:

$$
\begin{aligned}
& P T E_{2-\text { tier }}=P T E_{1-\text { tier }}+\sum_{i=1}^{n}\left(P T E_{12} \cdot P T E_{2 i, 4}\right) \\
& +\sum_{i, j=1}^{n}\left(P T E_{1,2 i} \cdot P T E_{2 i, 3 j} \cdot P T E_{3 j, 4}\right)
\end{aligned}
$$

5) Comparison: Comparing the expressions for PTE (1), (2), (5), (6) of the various configurations, the following order can be concluded:

$$
\begin{aligned}
& P_{T E} E_{4-\text { coil }, 2-\text { tier }}>P T E_{3-\text { coil }, 1-\text { tier }} \\
& \approx P T E_{3-\text { coil }}>P T E_{2-\text { coil }}
\end{aligned}
$$

\section{B. Electromagnetic Simulation Verification}

To verify the analytical expressions for PTEs and the comparison between the different schemes, 3D models are designed for each configuration and evaluated using a sixlayer head model in HFSS (ANSYS, Canonsburg, PA). Most importantly, the spatial distribution of the PTE across the $\mathrm{X}$ and $\mathrm{Y}$ axes is an essential parameter for the design of freely positioned implants. The simulation models used for the four configurations are shown in Fig. 2, annotated with coil positions. Simulated results of spatial distribution of PTE within $\pm 64 \mathrm{~mm}^{2}$ are included alongside each scheme.

The power coverage of the 2-coil and 3-coil configurations have a focused power profile, as shown in Fig 2(a.2,b.2). The maximum PTE is located at the central position of the Tx coil. The PTE degrades significantly as the Rx coild moves away from the centre of the Tx coil. The maximum PTE of the 3coil link here is twice that of the 2-coil link. The average PTE of 3-coil and 2-coil link can achieve to $4.67 \%$ and $0.44 \%$.
When the single resonant coil (i.e. in a 3-coil link) is replaced with an array of resonators, as shown in Fig. 2(c.2), the PTE can be evenly distributed over a increased range. To evaluate the PTE and uniformity of power coverage across the different schemes, the figure of merit (FoM) can be defined as: $F O M=\overline{P T E} \pm R S D$, where $\overline{P T E}$ the average value of PTE is expressed as: $\overline{P T E}=\sum_{i=0}^{N} P T E_{i} / N$. The relative standard deviation (RSD) of PTE can reveal the PTE fluctuations over a wide range. This is defined as:

$$
R S D=\sqrt{\frac{1}{N-1} \sum_{i=0}^{N}\left(P T E_{i}-\overline{P T E}\right)^{2}} / \overline{P T E}
$$

According to this expression, and the simulation results provided by HFSS, the FoM of each configuration is summarised in Table I. The essential requirements of wireless power transfer systems for multiple Rx devices are high PTE and low deviation in the spatial distribution of power. Simulation results confirm that the performance of 2-tier array significantly increases the average PTE to $11.9 \%$, whilst reducing the power distribution deviation to $1.77 \%$.

TABLE I

FOM FOR EACH INDUCTIVE LINK.

\begin{tabular}{c|cc}
\hline FoM & Average PTE & Power Distribution Deviation \\
\hline 2-Coil Link & $0.44 \%$ & $93.79 \%$ \\
3-Coil Link & $4.67 \%$ & $13.71 \%$ \\
1-Tier Resonator Array & $6.11 \%$ & $1.83 \%$ \\
2-Tier Resonator Array & $11.9 \%$ & $1.77 \%$ \\
\hline
\end{tabular}

\section{Self-Regulated Wireless Power And Data}

\section{A. System Implementation}

Fig. 3 shows the system architecture of the self-regulated power management unit. This uses a Schottky diode based full-wave rectifier, supply voltage monitor integrated with a bandgap voltage reference, a digital tuning capacitor array $C_{T}$, and finite state machine (FSM) for automatic tuning.

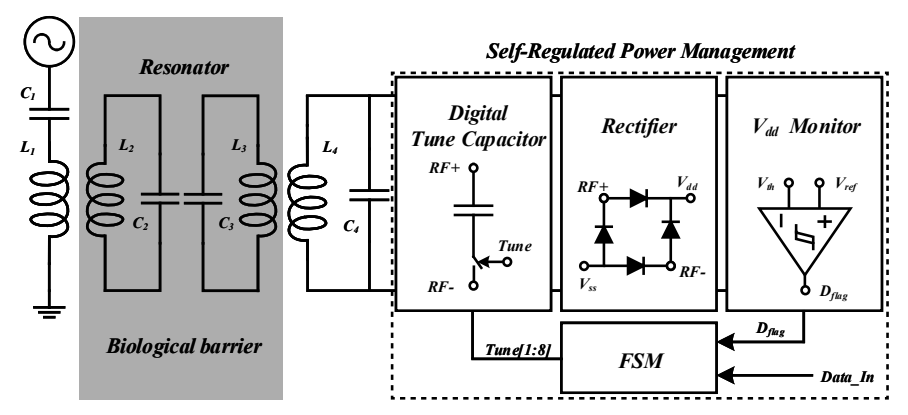

Fig. 3. Block diagram of the self-regulated power management unit.

This system operates as follows: the AC-DC conversion is first achieved using a full-wave rectifier. The rectified DC voltage $V_{d d}$ passes through a potential divider to define highand low-voltage thresholds $V_{t h}$. These threshold voltages are compared with the $1.24 \mathrm{~V}$ bandgap reference voltage $V_{\text {ref }}$, and generate flags $D_{\text {flag }}$ to represent whether the supply voltage is within the specified threshold range. The finite state machine (FSM) generates 8-bit tuning data based on these flags to control corresponding bits of the capacitor 
array $C_{T}$. The tuning capacitors $C_{4}, C_{T}$ and the Rx coil $L_{4}$ determine the self-resonant frequency (SRF) of the secondary LC tank. The voltage received on the secondary side can be effectively regulated by tuning its SRF because the entire inductive link is resonance-based. The resulting supply voltage $V_{d d}$ is finally checked using the over- and under-voltage flags generated by the supply voltage monitor. This self-regulated power management unit therefore ensures that the DC supply voltage remains within $1.7 \mathrm{~V}-1.9 \mathrm{~V}$ range.

\section{B. Self-Regulated Power Management}

1) Supply Voltage Monitor: The supply voltage monitor is designed to detect any fluctuation of the power supply voltage, and generate flags for the FSM to retune the system. This circuit is composed of a resistive divider, two strongarm comparators [31], shown in Fig. 4. The high $V_{d d(H)}$ and low supply voltage threshold $V_{d d(L)}$ are defined as: $V_{d d(H)}=V_{r e f}\left(R_{1}+R_{2}+R_{3}\right) / R_{3}=1.9 \mathrm{~V}$ and $V_{d d(L)}=$ $V_{\text {ref }}\left(R_{1}+R_{2}+R_{3}\right) /\left(R_{2}+R_{3}\right)=1.7 \mathrm{~V}$. When the supply voltage $V_{d d}$ is higher or lower than $V_{d d(H)}$ or $V_{d d(L)}$, the corresponding digital flags $D_{H}, D_{L}$ are set to 1 . Two digital flags generated by comparators are reset to low, When supply voltage $V_{d d}$ is adjusted within the thresholds $(1.7 \mathrm{~V}-1.9 \mathrm{~V})$.

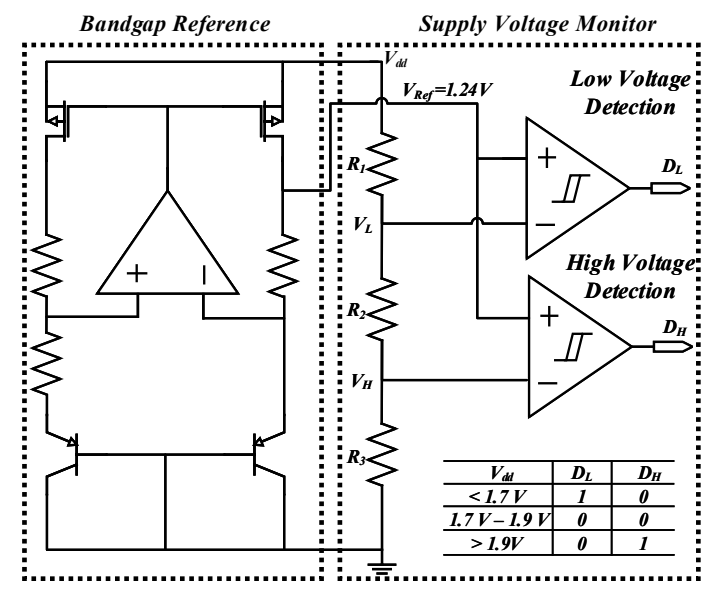

Fig. 4. Functional diagram of proposed supply voltage monitor.

2) Auto-Tune FSM: A simple four-state FSM is implemented to read and control the switches in the supply voltage monitor, and then to generate a 8-bit digital output for adding or removing capacitance from the secondary LC tank. The four states are idle, compare, tune and data. The FSM firstly ensure supply voltage fluctuations within the thresholds, and then the input data is transferred by modulating the power carrier through backscatter.

3) Digital Tune Capacitor: The unit capacitance of the 8 -bit binary-weighted capacitor array is $252.32 \mathrm{fF}$ (including parasitic capacitance), with a total capacitance of $9.08 \mathrm{pF}$. The SRF is inversely proportional to the square root of capacitance $f \propto 1 / \sqrt{C}$. SRF variations lead to induced voltage fluctuations on the secondary side. The maximum induced voltage is achieved when the secondary SRF is the same as the relay resonator. The induced voltage curve in terms of operating frequency conforms to the standard normal distribution. When the secondary SRF is close to the primary SRF, the unit capacitance may cause significant voltage changes in the

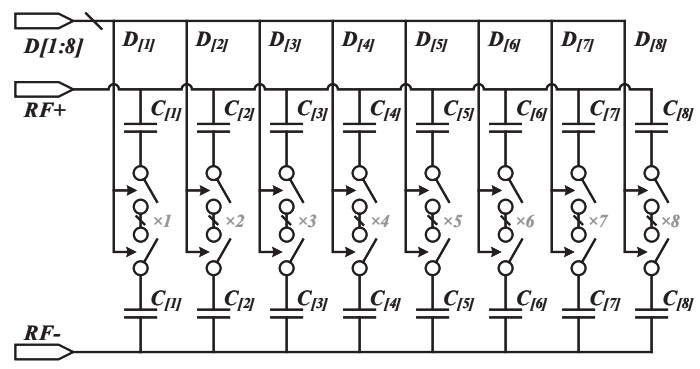

Fig. 5. Schematic of binary weighted digital tuned capacitor array.

induced secondary voltage. The initial value of the binaryweighted capacitor array is set to 128 to ensure sufficient tuning range from $381.89 \mathrm{MHz}$ to $509.99 \mathrm{MHz}$. According to the simulation results in Fig. 6, the rectified voltage variation is from $1.85 \mathrm{~V}$ to $2.31 \mathrm{~V}$. The self-regulated voltage can remain within $V_{d d(H)}$ and $V_{d d(L)}$ thresholds.

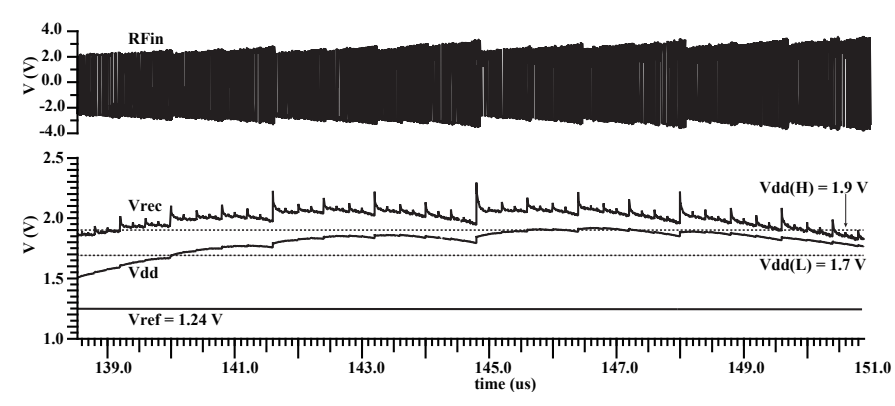

Fig. 6. Simulated results of self-regulated power management unit.

\section{Backscatter Data Communication}

Passive backscatter communication scheme consumes less power than active modulated communication schemes, such as UWB, BLE, as this passive modulation doesn't need to generate data carrier on-site. Here we use a $500 \mathrm{fF}$ capacitor to achieve $1.47 \%$ SRF offset, so that the transmitting energy can be backscattered from the secondary side to the primary side. At the same time, the voltage deviation caused on the secondary side is within $0.1 \mathrm{~V}$, which will not affect the stability of the supply voltage.

\section{CONCLusion AND Future Work}

This work has proposed 1- and 2-tier resonator coupled inductive links for simultaneously powering an arbitrary number of implant devices regardless of their specific location and/or orientation. The spatial power distribution has been analysed and compared against four different WPT schemes. This can guide the selection of a candidate WPT scheme for future implantable devices. Secondly, a circuit for self-regulating the power supply has been proposed. This can enable a distributed network of autonomous, independent implant nodes.

The proposed circuit has been designed and fabricated in a commercially-available $180 \mathrm{~nm}$ technology. Future work will integrate on-chip coils to form the secondary LC tank using stack die wire bonding [32]. The resonator array will be implemented using a flexible PCB substrate. The network will be validated using a software defined radio-frequency platform. This WIPT scheme will enable variety of multi-access systems to enable transmission wireless power transmission and data communication for multiple implants. 
bioRxiv preprint doi: https://doi.org/10.1101/2021.02.01.429239; this version posted February 2, 2021. The copyright holder for this preprint (which was not certified by peer review) is the author/funder, who has granted bioRxiv a license to display the preprint in perpetuity. It is made available under aCC-BY-NC-ND 4.0 International license.

\section{ACKNOWLEDGEMENT}

This work was supported by the Engineering and Physical Sciences Research Council (EPSRC) grant EP/M020975/1.

\section{REFERENCES}

[1] D. Seo, R. M. Neely, K. Shen, U. Singhal, E. Alon, J. M. Rabaey, J. M. Carmena, and M. M. Maharbiz, "Wireless recording in the peripheral nervous system with ultrasonic neural dust," Neuron, vol. 91, no. 3, pp. $529-539,2016$, [Online].

[2] M. L. Wang, S. Baltsavias, T. C. Chang, M. J. Weber, J. Charthad, and A. Arbabian, "Wireless data links for next-generation networked microimplantables," in 2018 IEEE Custom Integrated Circuits Conference (CICC), April 2018, pp. 1-9, [Online].

[3] J. Charthad, M. J. Weber, T. C. Chang, and A. Arbabian, "A mm-sized implantable medical device (IMD) with ultrasonic power transfer and a hybrid bi-directional data link," IEEE Journal of Solid-State Circuits, vol. 50, no. 8, pp. 1741-1753, Aug 2015, [Online].

[4] T. Maleki, N. Cao, S. H. Song, C. Kao, S. Ko, and B. Ziaie, "An ultrasonically powered implantable micro-oxygen generator (IMOG)," IEEE Transactions on Biomedical Engineering, vol. 58, no. 11, pp. 3104-3111, Nov 2011, [Online].

[5] C. Kim, J. Park, S. Ha, A. Akinin, R. Kubendran, P. P. Mercier, and G. Cauwenberghs, "A $3 \mathrm{~mm} \times 3 \mathrm{~mm}$ fully integrated wireless power receiver and neural interface system-on-chip," IEEE Transactions on Biomedical Circuits and Systems, pp. 1-1, 2019, [Online].

[6] S. A. Mirbozorgi, P. Yeon, and M. Ghovanloo, "Robust wireless power transmission to $\mathrm{mm}$-sized free-floating distributed implants," IEEE Transactions on Biomedical Circuits and Systems, vol. 11, no. 3, pp. 692-702, June 2017, [Online].

[7] J. Lee, F. Laiwalla, J. Jeong, C. Kilfoyle, L. Larson, A. Nurmikko, S. Li, S. Yu, and V. W. Leung, "Wireless power and data link for ensembles of sub-mm scale implantable sensors near 1GHz," in 2018 IEEE Biomedical Circuits and Systems Conference (BioCAS), Oct 2018, pp. 1-4, [Online].

[8] P. Troyk, S. Bredeson, S. Cogan, M. Romero-Ortega, S. Suh, Z. Hu, A. Kanneganti, R. Granja-Vazquez, J. Seifert, and M. Bak, "In-vivo tests of a 16-channel implantable wireless neural stimulator," in 2015 7th International IEEE/EMBS Conference on Neural Engineering (NER), April 2015, pp. 474-477, [Online].

[9] S.-I. Chang, S.-Y. Park, and E. Yoon, "Minimally-invasive neural interface for distributed wireless electrocorticogram recording systems," Sensors, vol. 18, no. 1, 2018, [Online].

[10] A. Khalifa, Y. Liu, Y. Karimi, Q. Wang, A. Eisape, M. Stanaćević, N. Thakor, Z. Bao, and R. Etienne-Cummings, "The microbead: A $0.009 \mathrm{~mm} 3$ implantable wireless neural stimulator," IEEE Transactions on Biomedical Circuits and Systems, vol. 13, no. 5, pp. 971-985, Oct 2019, [Online].

[11] J. S. Ho, A. J. Yeh, E. Neofytou, S. Kim, Y. Tanabe, B. Patlolla, R. E. Beygui, and A. S. Y. Poon, "Wireless power transfer to deep-tissue microimplants," Proceedings of the National Academy of Sciences, vol. 111, no. 22, pp. 7974-7979, 2014, [Online].

[12] S. I. Park, D. S. Brenner, G. Shin, C. D. Morgan, B. A. Copits, H. U. Chung, M. Y. Pullen, K. N. Noh, S. Davidson, S. J. Oh, J. Yoon, K.-I. Jang, V. K. Samineni, M. Norman, J. G. Grajales-Reyes, S. K. Vogt, S. S. Sundaram, K. M. Wilson, J. S. Ha, R. Xu, T. Pan, T.-i. Kim, Y. Huang, M. C. Montana, J. P. Golden, M. R. Bruchas, R. W. Gereau, and J. A. Rogers, "Soft, stretchable, fully implantable miniaturized optoelectronic systems for wireless optogenetics," Nature Biotechnology, vol. 33, no. 12, pp. 1280-1286, Dec 2015, [Online].

[13] L. B. Leene, M. Maslik, P. Feng, K. M. Szostak, F. Mazza, and T. G. Constandinou, "Autonomous SoC for neural local field potential recording in mm-scale wireless implants," in 2018 IEEE International Symposium on Circuits and Systems (ISCAS), May 2018, pp. 1-5, [Online].

[14] P. Feng, M. Maslik, and T. G. Constandinou, "EM-lens enhanced power transfer and multi-node data transmission for implantable medical devices," in 2019 IEEE Biomedical Circuits and Systems Conference (BioCAS), Oct 2019, pp. 1-4, [Online].

[15] S. Lee, A. J. Cortese, A. P. Gandhi, E. R. Agger, P. L. McEuen, and A. C. Molnar, "A $250 \mu \mathrm{m} \times 57 \mu \mathrm{m}$ microscale opto-electronically transduced electrodes (MOTEs) for neural recording," IEEE Transactions on Biomedical Circuits and Systems, vol. 12, no. 6, pp. 1256-1266, 2018, [Online].
[16] M. Mujeeb-U-Rahman, D. Adalian, C.-F. Chang, and A. Scherer, "Optical power transfer and communication methods for wireless implantable sensing platforms," Journal of Biomedical Optics, vol. 20, no. 9, pp. 1 $-9,2015$, [Online].

[17] R. Das, F. Moradi, and H. Heidari, "Biointegrated and wirelessly powered implantable brain devices: A review," IEEE Transactions on Biomedical Circuits and Systems, pp. 1-1, 2020, [Online].

[18] D. Ahn and M. Ghovanloo, "Optimal design of wireless power transmission links for millimeter-sized biomedical implants," IEEE Transactions on Biomedical Circuits and Systems, vol. 10, no. 1, pp. 125-137, Feb 2016, [Online].

[19] M. Zargham and P. G. Gulak, "Fully integrated on-chip coil in 0.13 $\mu \mathrm{m}$ CMOS for wireless power transfer through biological media," IEEE Transactions on Biomedical Circuits and Systems, vol. 9, no. 2, pp. 259271, April 2015, [Online].

[20] M. Mark, T. Björninen, L. Ukkonen, L. Sydänheimo, and J. M. Rabaey, "SAR reduction and link optimization for $\mathrm{mm}$-size remotely powered wireless implants using segmented loop antennas," in 2011 IEEE Topical Conference on Biomedical Wireless Technologies, Networks, and Sensing Systems, Jan 2011, pp. 7-10, [Online].

[21] M. Schormans, V. Valente, and A. Demosthenous, "Practical inductive link design for biomedical wireless power transfer: A tutorial," IEEE Transactions on Biomedical Circuits and Systems, vol. 12, no. 5, pp. 1112-1130, 2018, [Online].

[22] J. Lee, E. Mok, J. Huang, L. Cui, A.-H. Lee, V. Leung, P. Mercier, S. Shellhammer, L. Larson, P. Asbeck et al., "An implantable wireless network of distributed microscale sensors for neural applications," in 2019 9th International IEEE/EMBS Conference on Neural Engineering (NER). IEEE, 2019, pp. 871-874, [Online].

[23] N. Ahmadi, M. L. Cavuto, P. Feng, L. B. Leene, M. Maslik, F. Mazza, O. Savolainen, K. M. Szostak, C.-S. Bouganis, J. Ekanayake et al., "Towards a distributed, chronically-implantable neural interface," in 2019 9th International IEEE/EMBS Conference on Neural Engineering (NER). IEEE, 2019, pp. 719-724, [Online].

[24] M. M. Ghanbari, D. K. Piech, K. Shen, S. F. Alamouti, C. Yalcin, B. C. Johnson, J. M. Carmena, M. M. Maharbiz, and R. Muller, "A sub- $\mathrm{mm}^{3}$ ultrasonic free-floating implant for multi-mote neural recording," IEEE Journal of Solid-State Circuits, vol. 54, no. 11, pp. 3017-3030, 2019, [Online].

[25] Y. Urzhumov and D. R. Smith, "Metamaterial-enhanced coupling between magnetic dipoles for efficient wireless power transfer," Phys. Rev. $B$, vol. 83, p. 205114, May 2011, [Online].

[26] J. Lee, F. Laiwalla, J. Jeong, C. Kilfoyle, L. Larson, A. Nurmikko, S. Li, S. Yu, and V. W. Leung, "Wireless power and data link for ensembles of sub-mm scale implantable sensors near 1ghz," in 2018 IEEE Biomedical Circuits and Systems Conference (BioCAS). IEEE, 2018, pp. 1-4, [Online].

[27] R. Biran, D. C. Martin, and P. A. Tresco, "The brain tissue response to implanted silicon microelectrode arrays is increased when the device is tethered to the skull," Journal of Biomedical Materials Research Part A, vol. 82, no. 1, pp. 169-178, 2007, [Online].

[28] S. S. Ghoreishizadeh, D. Haci, Y. Liu, N. Donaldson, and T. G. Constandinou, "Four-wire interface asic for a multi-implant link," IEEE Transactions on Circuits and Systems I: Regular Papers, vol. 64, no. 12, pp. 3056-3067, 2017, [Online].

[29] P. Feng and T. G. Constandinou, "Robust wireless power transfer to multiple mm-scale freely-positioned neural implants," in 2018 IEEE Biomedical Circuits and Systems Conference (BioCAS), 2018, pp. 14, [Online].

[30] M. Kiani and M. Ghovanloo, "The circuit theory behind coupledmode magnetic resonance-based wireless power transmission," IEEE Transactions on Circuits and Systems I: Regular Papers, vol. 59, no. 9, pp. 2065-2074, Sep. 2012, [Online].

[31] B. Razavi, "The StrongARM latch [A Circuit for All Seasons]," IEEE Solid-State Circuits Magazine, vol. 7, no. 2, pp. 12-17, Spring 2015, [Online].

[32] P. Feng, P. Yeon, Y. Cheng, M. Ghovanloo, and T. G. Constandinou, "Chip-scale coils for millimeter-sized bio-implants," IEEE Transactions on Biomedical Circuits and Systems, vol. 12, no. 5, pp. 1088-1099, 2018, [Online]. 